\section{Kidney \\ Blood Pressure Research}

Kidney Blood Press Res 2016;41:450-459

DOI: 10.1159/000443446

Published online: July 15, 2016

Accepted: May 18, 2016

\title{
Co-inhibition of Angiotensin II Receptor and Endothelin-1 Attenuates Renal Injury in Unilateral Ureteral Obstructed Mice
}

\author{
Yoon-Kyung Chang a,b Hyunsu Choic Jin Young Jeongd,e Ki-Ryang Na ${ }^{\mathrm{e}, \mathrm{f}}$ \\ Kang Wook Lee $e^{e, f}$ Dae Eun Choi ${ }^{, f}$
}

aDepartment of Nephrology, Daejeon St. Mary Hospital, Daejeon; 'Department of Nephrology, College of Medicine, The Catholic University of Korea, Seoul; 'Clinical Research Institute, Daejeon St.

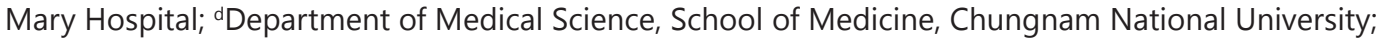
eDepartment of Nephrology, Chungnam National University Hospital; fDepartment of Nephrology, School of Medicine, Chungnam National University, Daejeon, Korea

\section{Key Words}

Endothelin-1 • Renin-angiotensin system • Fibrosis • Ureteral obstruction • Inflammation

\begin{abstract}
Background/Aims: Both endothelin-1 (ET-1) and the renin-angiotensin system (RAS) may play important roles in renal fibrosis in the obstructed kidney. However, there have been few clear demonstrations of a relationship between their activation and additive or synergistic roles in renal fibrosis. We investigated the protective roles and relationship between renal RAS and ET-1 in unilateral ureteral obstruction (UUO) mice. Methods: 8-week-old male C57BL/6 mice were divided into seven groups: sham, bosentan+sham, valsartan+sham, vehicle+UUO, bosentan+UUO, valsartan+UUO, and valsartan+bosentan+UUO. Valsartan and bosentan were administered orally using an NG tube (valsartan $10 \mathrm{mg} / \mathrm{kg} /$ day, bosentan $100 \mathrm{mg} / \mathrm{kg} /$ day for 8 days, after which the molecular and structural kidney parameters were evaluated. Bosentan treatment elevated plasma renin activity, renal renin, and AT1R expression in UUO mice. Results: Although valsartan decreased plasma ET-1 in these mice, it did not affect ET(A) or $\mathrm{ET}(\mathrm{B})$ in their kidneys. Co-treatment with valsartan and bosentan decreased ET-1 in these mice compared to the single treatments. Bosentan, but not valsartan, elevated eNOS expression in their kidneys. Co-treatment with valsartan and bosentan reduced TGF- $\beta, \alpha-S M A$, and collagen IV expression, and the Masson's trichrome stained area in their kidneys. Conclusions: Bosentan and valsartan acted complementarily, and co-treatment with both drugs had an additive protective effect against renal fibrosis.
\end{abstract}




\section{Kidney Blood Pressure Research}

Chang et al.: Antifibrotic Effect of Valsartan and Bosentan

\section{Introduction}

Renal inflammation and fibrosis are major pathomechanisms of chronic kidney disease, which requires renal replacement therapy, including dialysis and kidney transplantation [1]. Unilateral ureteral obstruction (UUO) is a well-known model for renal inflammation and fibrosis [2]. In the UUO kidney, pressure-induced tubular cell injury, activation of RAS, and various inflammation- and fibrosis-inducing proteins such as MCP- 1 and TGF- $\beta$ induce the infiltration of macrophages and fibroblasts, leading to renal inflammation and fibrosis [3].

The renin-angiotensin system (RAS) plays a key role in the development and progression of renal inflammation and fibrosis in UUO models [4-6]. RAS inhibition using an angiotensinconverting enzyme (ACE) inhibitor and an angiotensin II receptor blocker (ARB) has been shown to reduce renal inflammation and fibrosis in various animal models of chronic renal injury [7, 8]. In addition, human studies have shown that inhibition of RAS by an ACE inhibitor and ARB reduces albuminuria (including inflammation and fibrosis), a marker of chronic renal injury, in patients with diabetic nephropathy or hypertension $[9,10]$.

Endothelin-1 (ET-1) is a potent vasoconstrictor, which binds two types of receptors, ET(A) and ET(B), and plays an important role in various renal injuries, including renal tubular necrosis, interstitial inflammation, and renal fibrosis. Inhibition of ET-1 by bosentan, an ET(A) and ET(B) receptor antagonist, has favorable protective effects in acute and chronic renal injuries induced by glycerol-induced myoglobinuria, radiocontrast, the remnant kidney model, and the ischemia-reperfusion injury model [11-15].

Inhibition of ET-1 and RAS may have additive effects against kidney injury. For example, AT1-deficient mice show elevated ET-1 expression in tubular cells, suggesting that both inhibition of AT1 and ET-1 may have additive roles in various kidney injuries [16]. Yang et al. reported that bosentan activates the renin-angiotensin system in the blood and kidney tissue of mice, indicating that inhibition of both ET1 and RAS may have additive effects in the control of hypertension [17]. Gomez et al. reported that bosentan reduced renal inflammatory markers in angiotensin II-treated rats, suggesting that inhibition of both RAS and ET-1 may have additional effects in a renal inflammatory injury model [18]. Although some reports have shown renoprotective effects in an acute ischemia-reperfusion injury mouse model and an aristolochic acid-induced nephropathy model, other reports have suggested no additive effect [19-22].

Here, we evaluated the relationship between RAS and ET-1, and the associated mechanism for reducing renal fibrosis. We also investigated whether inhibition of both RAS and ET-1 showed additional improvement in inflammation or fibrosis in a mouse model of UUO.

\section{Materials and Methods}

\section{Animals and drugs}

All of the experiments were performed using 8-week-old male C57BL/6 mice, weighing 22-25 g (Samtako, Kyoung Gi-Do, Korea). The mice were given a standard laboratory diet (Damul Science, Daejeon, Korea) and water, and were cared for according to a protocol approved by the Institutional Animal Care and Use Committee of the Catholic University (CMCDJ-AP-2014-001).

The mice were divided into seven groups: sham, bosentan+sham, valsartan+sham, UUO-vehicle, UUO-bosentan, UUO-valsartan, and UUO-combination therapy. Valsartan (10 mg/kg) and bosentan (100 $\mathrm{mg} / \mathrm{kg}$ ) were purchased commercially and given by oral gavage. Both valsartan and bosentan were treated once daily from 1 day preoperatively to 7 days postoperatively. As described previously [23], for the UUO operation, the mice were anesthetized with intraperitoneal ketamine $(2 \mathrm{mg} / \mathrm{kg}$, Ketalar; Bayer, Leverkusen, Germany) and xylazine ( $200 \mu \mathrm{L} / \mathrm{kg}$, Rompun; Bayer). First, a 1.5-2 cm midline abdominal incision was made using a sterile technique. The intestines were pushed gently medially. The left ureter was identified at the lower renal pole. An obstruction was created with double 5-0 silk sutures. The sham operation for control 


\section{Kidney Blood Pressure Research}

kidneys consisted of a similar midabdominal incision and visualization of the left ureter without further manipulation. The animals were sacrificed 7 days after the operation.

\section{Blood and tissue preparation}

Blood was collected from the inferior vena cava at sacrifice under anesthesia, and placed into prechilled microcentrifuge tubes $\left(4^{\circ} \mathrm{C}\right)$, after which $10 \mathrm{mM}$ disodium EDTA was added to prevent coagulation. The plasma was separated by centrifugation $\left(10 \mathrm{~min}, 4^{\circ} \mathrm{C}\right)$, and aliquots were snap-frozen in liquid nitrogen and stored at $-70^{\circ} \mathrm{C}$. Tissues were prepared as previously described [24]. Briefly, the left kidney was excised immediately after sacrifice and cut into three coronal sections. Two pieces of the kidney were snap-frozen in liquid nitrogen and kept at $-70^{\circ} \mathrm{C}$ for subsequent RNA extraction and protein analysis. The other portion of kidney was fixed in $10 \%$ buffered formaldehyde at room temperature and embedded in Paraplast (Sherwood Medical, St. Louis, MO, USA) for light microscopy and immunohistochemistry.

\section{Plasma renin activity and ET-1 measurements}

As described previously [23], plasma renin activity was measured by radioimmunoassay using a commercially available kit, according to the manufacturer's protocol. Each plasma sample was divided into two parts: one incubated at $37^{\circ} \mathrm{C}$ for $2 \mathrm{~h}$ and one incubated at $4^{\circ} \mathrm{C}$ for $2 \mathrm{~h}$. Standards contained angiotensin I at concentrations of $0.0,0.2,0.8,2,8$, and $20 \mathrm{ng} / \mathrm{mL}$. After adding $100 \mu \mathrm{L}$ tracer-buffer reagent to each $37^{\circ} \mathrm{C}$ and $4^{\circ} \mathrm{C}$ plasma sample, as well as the standard, the tubes were incubated at room temperature for 3 $\mathrm{h}$ and decanted. Radioactivity was measured for $1 \mathrm{~min}$ in a counter. Renin activity was calculated from the standard graph, and the values of the $4^{\circ} \mathrm{C}$ samples were subtracted from those of the $37^{\circ} \mathrm{C}$ samples. Renin activity is expressed in $\mathrm{ng} / \mathrm{mL}$ per hour. Plasma ET-1 levels were measured using an ELISA kit (R\&D Systems, Minneapolis, MN, USA) according to the manufacturer's protocol with certain reagents (nos. WA126, DY994, DY995, and DY999, R\&D Systems). Values are shown as ng renin per mL plasma.

\section{Real-time reverse transcription PCR}

Total RNA was prepared from kidney tissues using the TRIzol reagent (Invitrogen, Carlsbad, CA, USA). cDNA was synthesized from $1 \mu \mathrm{g}$ RNA using Reverse Transcriptase Premix (Elpis Biotech, Daejeon, Korea). After reverse transcription, the cDNA was used as a template in PCR reactions using gene-specific primer pairs. cDNA was amplified in a Power SYBR Green PCR Master Mix (Applied Biosystems, Warrington, UK). Quantitative real-time PCR was performed with an ABI 7500 FAST (Applied Biosystems, Foster City, CA, USA). The relative levels of mRNA were normalized to glyceraldehyde-3-phosphate dehydrogenase (GAPDH). The sequences of the primer sets were as follows: renin, $5^{\prime}$-TGG GTG CCC TCC ACC AAG TG-3' (sense) and $5^{\prime}$-CTC CCA GGG CTT GCA TGA TCA-3' (antisense); AT1R, $5^{\prime}$-AAC AGC TTG GTG GTG ATC GTC-3' (sense) and $5^{\prime}$-CAT AGC GGT ATA GAC AGC CCA-3' (antisense); ET(A)R, 5'- GGT GGC TCT TTG GGT TCT-3' (sense) and 5'-GAC GCT GTT TGA GGT GCT-3' (antisense); ET(B)R, $5^{\prime}$-TGC GAA ATG CTC AGG AAG-3' (sense) and $5^{\prime}$-ACG AGG ACC AGG CAG AAG-3' (antisense); and ET-1, 5' ${ }^{\prime}$-CCT GGA CAT CAT CTG GGT C-3' (sense) and $5^{\prime}$-TGT GGC CTT ATT GGG AAG-3' (antisense).

\section{Western blot analysis}

The expression of TGF- $\beta$, $\alpha$-SMA, collagen IV, iNOS, and eNOS proteins in kidney tissue was analyzed by Western blotting as described previously [25], Briefly, equal amounts of protein samples were resolved by SDS-PAGE. The proteins were electroblotted onto a nitrocellulose membrane (Amersham, Piscataway, $\mathrm{NJ}$, USA). Then the membranes were incubated overnight at $4^{\circ} \mathrm{C}$ with antibodies against TGF- $\beta$ (Abcam, Cambridge, UK), $\alpha$-SMA (Abcam), collagen IV (Abcam), iNOS (Santa Cruz, CA, USA), or eNOS (Santa Cruz), followed by incubation with a secondary antibody.

\section{Light microscopy}

Pieces of kidney embedded in paraffin wax were cut into $4 \mu \mathrm{m}$ sections and mounted on glass slides. The sections were deparaffinized with xylene, stained with hematoxylin and eosin (H\&E) and Masson's trichrome, and examined under an Olympus BX51 microscope (Tokyo, Japan). The tubulointerstitial injury score was evaluated based on morphological changes in the tubules, such as dilation, distortion of tubular basement membranes, and atrophy, as follows: grade 0 , no morphological deformities; grade $1,<10 \%$; 


\section{Kidney Blood Pressure Research}

Chang et al.: Antifibrotic Effect of Valsartan and Bosentan

grade 2, <25\%; grade 3, <50\%; grade 4, <75\%; and grade $5, \geq 75 \%$ involved. Ten consecutive fields were examined under $400 \times$ magnification and averaged per slide. Two pathologists examined and scored the kidney sections in a blinded fashion. Renal fibrotic areas were quantified by morphometric analysis using a light microscope and a digital camera-based image analyzer (Metamorpho, ver. 4.6; Olympus, Tokyo, Japan). Blue-stained (fibrotic) areas were quantified by computer-based morphometric analysis.

Immunohistochemistry

Immunohistochemistry was performed as described previously [24]. Paraffin wax-embedded tissues were cut into $4 \mu \mathrm{m}$ sections, mounted on glass slides, and stained using indirect immunoperoxidase. The slides were processed for identification of myofibroblast differentiation (anti- $\alpha$-SMA antibody; Abcam) and TGF- $\beta$ expression (anti-TGF- $\beta$ antibody; Abcam), followed by detection using diaminobenzidine (Sigma Chemical Co., St. Louis, MO, USA). All of the samples were evaluated under an Olympus BX51 microscope. The areas stained for $\alpha$-SMA and TGF- $\beta$, as percentages of the total area in 10 different fields of each section under 200x magnification, were determined automatically using a digital camera-based image analyzer (Metamorpho, ver. 4.6).

\section{Statistical analysis}

Data are reported as means \pm SD. Multiple comparisons among groups were performed using one-way ANOVA with a post hoc Bonferroni test correction (SPSS, ver. 11.0 for Windows; SPSS, Inc., Chicago, IL, USA). A difference between groups was considered significant at $\mathrm{P}<0.05$.

\section{Results}

Effects of ET1 inhibition on RAS activation in sham and UUO kidneys

Treatment with bosentan increased the mRNA expression of renin in sham and UUO kidneys, and elevated the mRNA levels of AT1R in sham kidneys. However, there were no significant differences in mRNA expression of AT1R in UUO kidneys (Fig. 1). Valsartan treatment did not affect renal ET(A)R or ET(B)R mRNA expression in UUO kidneys. Bosentan and valsartan significantly reduced the mRNA expression of ET-1 compared to vehicletreated UUO mice. Co-treatment of bosentan and valsartan significantly reduced the plasma levels of ET-1 compared to bosentan-treated or valsartan-treated UUO mice. Plasma renin activity (PRA) was higher in UUO mice than in sham mice, and plasma renin activity (PRA) was higher in valsartan-treated and bosentan-treated UUO mice compared to vehicle-treated UUO mice (Fig. 1).

\section{Effects of bosentan on iNOS and eNOS}

UUO decreased the expression of eNOS and iNOS in vehicle-treated mice. Bosentan treatment increased eNOS expression in UUO kidneys. In addition, co-treatment with bosentan and valsartan increased eNOS expression in UUO kidneys. Although valsartan treatment increase iNOS expression in UUO kidney, there were no statistical differences. Also bosentan did not affect the iNOS expression in UUO kidney (Fig. 2).

\section{Effects of co-treatment with valsartan and bosentan on renal injury}

UUO kidneys showed tubular dilation and necrosis and tubule interstitial inflammation. Bosentan and valsartan treatment significantly reduced renal injury compared to the vehicle-treated UUO kidney. Co-treatment with bosentan and valsartan showed decreased renal damage compared to bosentan-treated or valsartan-treated UUO kidneys (Fig. 3).

\section{Effects of co-treatment with bosentan and valsartan on renal fibrosis}

Masson's trichrome staining showed that UUO aggravated renal interstitial fibrosis, which was attenuated by bosentan and valsartan treatment, preserving tubular morphology. Compared to single treatments, co-treatment with bosentan and valsartan 


\section{Kidney Blood Pressure Research}

Fig. 1. Relationship between ET-1 and RAS. Bosentan treatment increased the mRNA expression of renin in sham and UUO kidneys (A). It also elevated the mRNA expression of AT1R in sham kidneys. However, there were no significant differences in mRNA expression of AT1R in UUO kidneys (B). Valsartan treatment did not affect renal ET(A)R or ET(B)R mRNA expression in UUO kidneys ( $\mathrm{C}$ and D). Bosentan and valsartan significantly reduced renal ET-1 mRNA expression and plasma levels of ET-1 compared to vehicle-treated UUO mice. Co-treatment with bosentan and valsartan significantly reduced renal ET-1 mRNA expression and plasma levels of ET-1 compared to single-treated UUO mice (E and F). Plasma renin activity (PRA) was higher in UUO mice than in sham mice. Plasma renin activity (PRA) was higher in valsartan- and bosentan-treated UUO mice compared to vehicle-treated UUO mice (G). ${ }^{*} P<0.05$, vs. sham, $\# P<0.05$, vs. UUO, $† P<0.05$, vs. single treatment in UUO.

showed a decreased area of blue stain in the UUO kidney (Fig 3). The renal expression of TGF- $\beta, \alpha-S M A$, and collagen IV significantly increased in UUO mice. Bosentan and valsartan treatment decreased the renal expression of TGF- $\beta, \alpha$-SMA, and collagen IV in UUO kidneys. Co-treatment with bosentan and valsartan further decreased the renal expression of TGF- $\beta$, $\alpha$-SMA, and collagen IV, compared to UUO kidney groups treated with bosentan or valsartan alone (Fig. 4). The areas stained with TGF- $\beta$ and $\alpha$-SMA showed similar patterns to the results of the immunoblotting (Fig. 3). 


\section{Kidney Blood Pressure Research}

Fig. 2. Representative kidney tissue sections immunostained for eNOS and iNOS. UUO kidneys showed decreased expression of eNOS and iNOS in vehicle-treated mice. Bosentan treatment increased eNOS expression in the kidneys. In addition, co-treatment of bosentan and valsartan increased eNOS expression. \# $P<0.05$, vs. UUO.

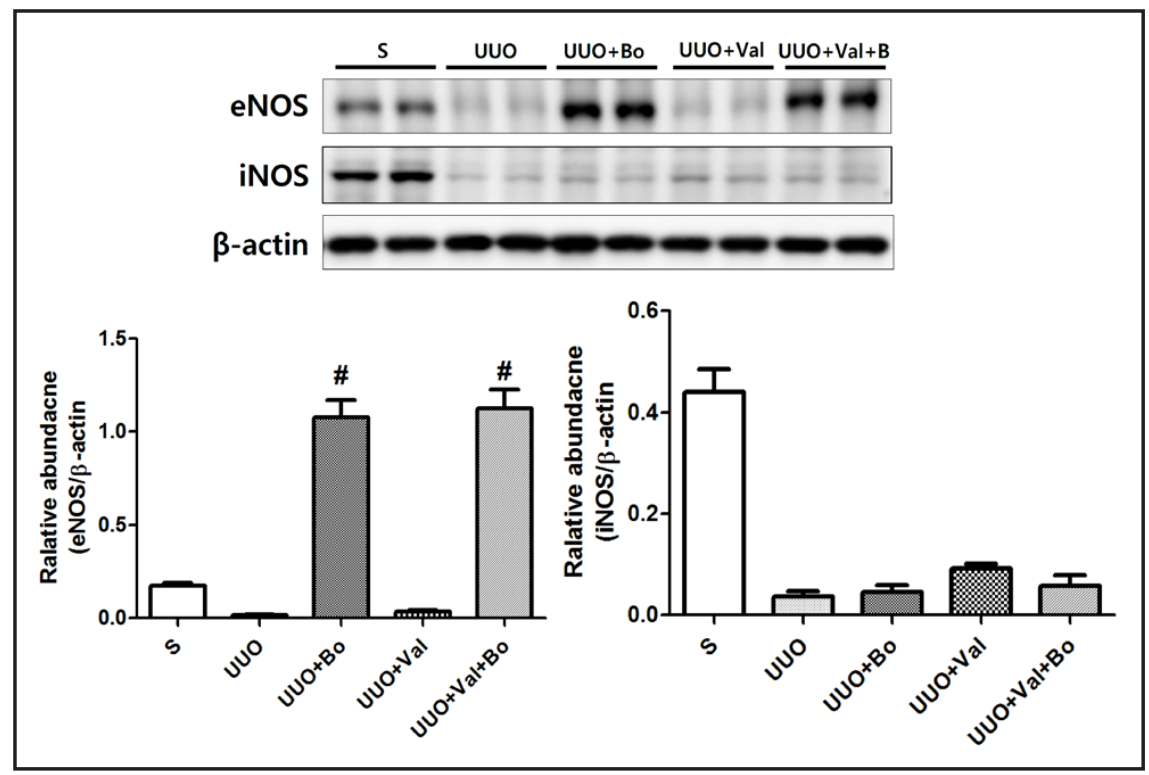

\section{Discussion}

Our study demonstrates that co-treatment with bosentan and valsartan has additive protective effects in UUO-induced renal fibrosis. Although there have been reports that inhibition of both ET-1 and RAS has favorable effects on renal hemodynamics, and antiproteinuric and anti-inflammatory effects in various kidney injuries, there is little evidence clearly demonstrating any relationship between ET-1 and RAS in these effects against renal fibrosis $[18,19]$. In addition, some studies have reported that inhibition of ET-1 did not decrease renal fibrosis in streptozotocin-induced transgenic rats and subtotally nephrectomized rats $[21,22]$. There are also confusing results on the relationship between RAS and ET-1 in various kidney injury models. AT-1-deficient mice show upregulation of ET-1 in tubular epithelial cells [16]. Lariviere et al. reported that RAS inhibition by an angiotensin II receptor blocker, but not an ACE inhibitor, reduced ET-1 production in a rat remnant kidney model [13]. The authors suggested that AT-1-specific inhibition may help reduce ET-1 generation. Lehrke et al. reported that ACE inhibitors decreased ET(B) receptor expression in the kidneys of patients with chronic kidney disease [26]. However, Moridaira et al. reported that enalapril treatment reduced ET-1 expression and increased ET(B) receptor expression in UUO kidneys [27]. Bosentan treatment increases the levels of plasma renin activity and renin expression in the mouse kidney [17]. In addition, CYP1A1-Ren-2 transgenic rats, an angiotensin II overproduction-induced hypertension model, show no response to ET-1 inhibition [28].

In this study, bosentan treatment increased plasma renin activity, and renal renin and AT1 receptor mRNA expression in UUO mice. Valsartan treatment decreased plasma ET-1 and ET-1 renal mRNA expression in these mice, but did not affect renal ET(A) or ET(B) receptor expression. In addition, co-treatment with valsartan and bosentan further decreased renal and plasma ET-1 mRNA expression compared to the single treatments. These results suggest that RAS activation by bosentan and RAS inhibition by valsartan have a counterbalanced effect in lowering ET-1.

It has been suggested that NO may play a major role in acute and chronic kidney injury $[25,29,30]$. Spontaneous NO donors show reno-protective effects in post-ischemic AKI, cisplatin nephrotoxicity, and UUO kidneys [31-33]. In particular, NO donors suppress TGF- $\beta$ expression and renal fibrosis in UUO kidneys [33]. Strong NO blockade by L-NAME diminishes the reno-protective effect of co-inhibiting ET-1 and RAS. However, mild NO inhibition 


\section{Kidney Blood Pressure Research}

Fig. 3. Representative kidney tissue sections with H\&E staining (A), Masson's trichrome staining (B), and immunostaining for TGF- $\beta(C)$, and $\alpha$-SMA (D). (A) Bosentan and valsartan treatment significantly reduced renal injury scores in UUO kidneys. Co-treatment with bosentan and valsartan reduced renal injury scores significantly compared to single treatments (B) The blue-stained fibrotic area of the kidney was greater in vehicle-treated UUO mice than in sham mice, and co-treatment with bosentan and valsartan reduced the fibrotic area significantly in UUO kidneys compared to single treatments. The TGF- $\beta$-positive area (dark brown, C) and $\alpha$-SMA-positive area (dark brown, D) of the kidney were greater in vehicle-treated UUO mice than in sham mice. Bosentan and valsartan treatment significantly reduced the $\alpha$-SMAand TGF- $\beta$-positive areas in UUO kidneys, as did co-treatment, compared to single treatments. $* P<0.05$, vs. Sham, \# $P<0.05$, vs. UUO, $\dagger P<0.05$, vs. single treatment in UUO, original magnification $\times 200$, scale bar $=100 \mu \mathrm{m}$.
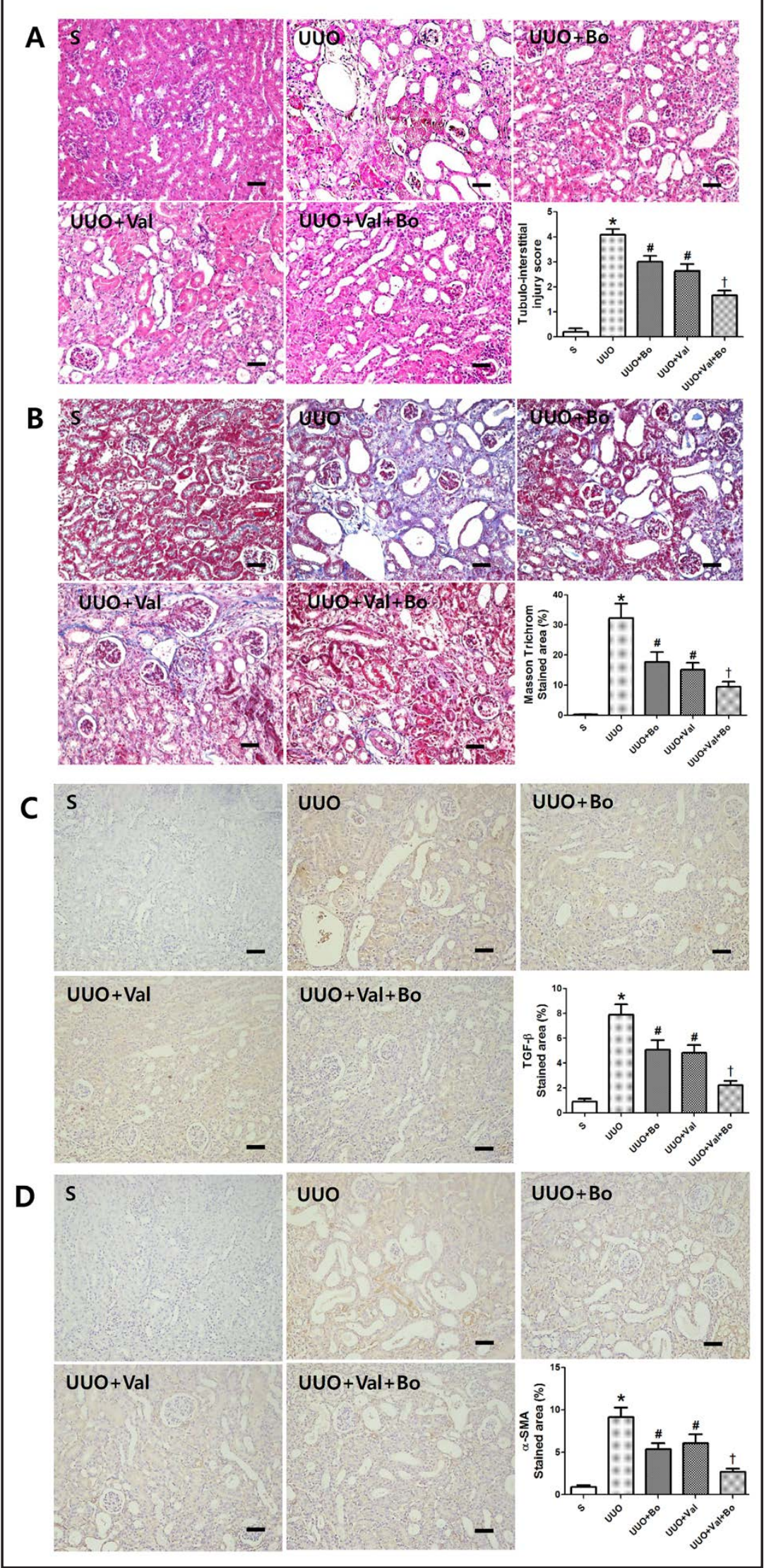


\section{Kidney Blood Pressure Research}

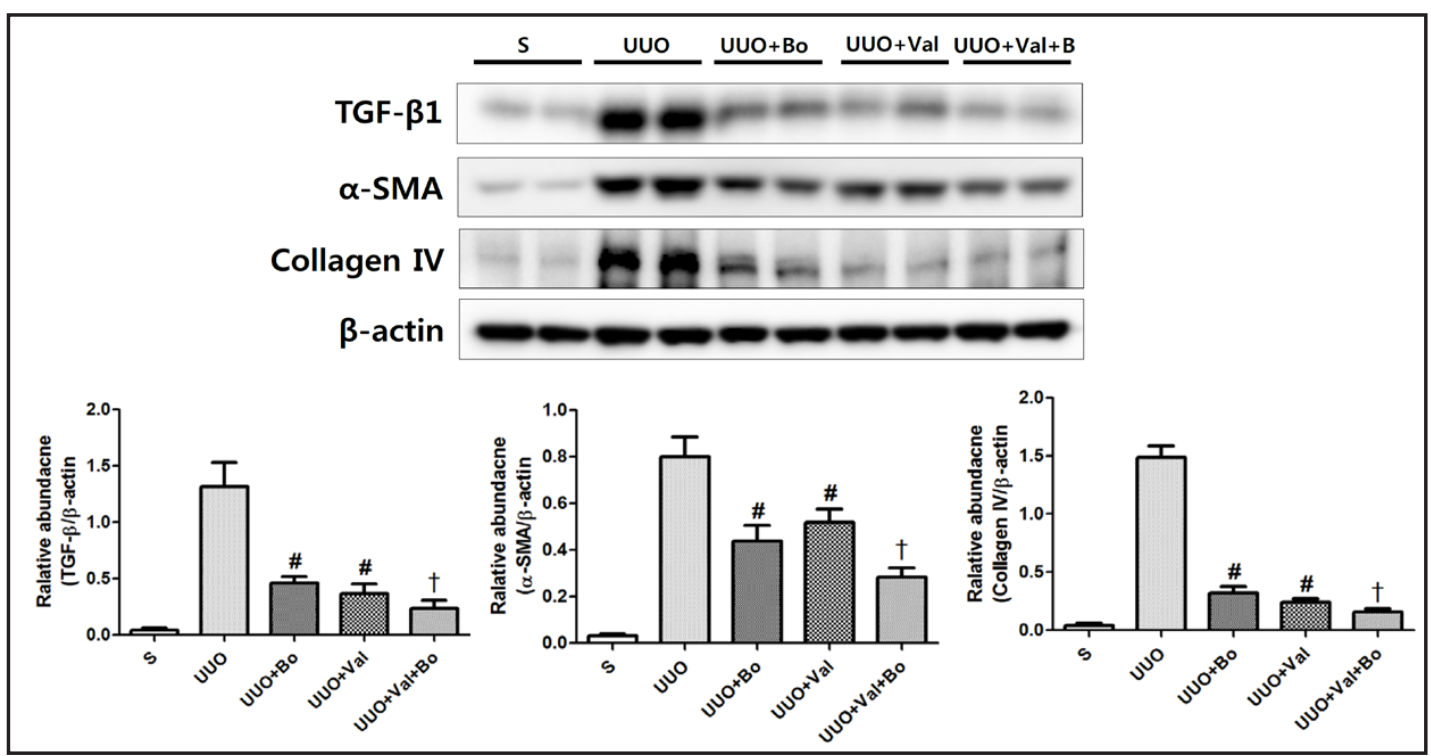

Fig. 4. Representative kidney tissue sections immunostained for TGF- $\beta, \alpha$-SMA, and collagen IV. The levels of TGF- $\beta, \alpha$-SMA, and collagen IV were greater in vehicle-treated UUO mice than in sham mice, and bosentan and valsartan treatment significantly reduced the levels of each in UUO kidneys $(\mathrm{N})$, as did co-treatment, compared to single treatments. \# $P<0.05$, vs. UUO, $\uparrow P<0.05$, vs. single treatment in UUO.

enhances the reno-protective effect of co-inhibiting ET-1 and RAS [19]. In endothelial cells, ET-1 suppresses eNOS expression, resulting in decreased NO generation. In addition, bosentan treatment elevates eNOS expression [34]. ET-1 and L-NAME can induce TGF- $\beta$ expression in the kidney [35]. There are conflicting data on the effects of RAS inhibition on NOS expression in kidney injury. In a study that used a nephrectomy kidney model, an ACE inhibitor elevated iNOS expression [36]. However, in another study, losartan decreased iNOS expression in UUO kidneys [37].

\section{Conclusion}

In our study, bosentan elevated renal eNOS expression in UUO kidneys, and valsartan minimally elevated its expression. Elevated renal eNOS expression by bosentan may improve renal fibrosis in UUO kidneys by reducing TGF- $\beta$ expression, because TGF- $\beta$ expression is a key factor involved in the progression of interstitial fibrosis in UUO-induced renal injuries.

Angiotensin II acts in an autocrine and/or a paracrine manner to stimulate TGF- $\beta$ expression in the kidney. In addition, we found that inhibition of RAS reduced TGF- $\beta$ expression in the UUO kidney. Both the increase in eNOS due to bosentan and inhibition of angiotensin II action by valsartan may additively reduce TGF- $\beta$ expression, resulting in decreased renal fibrosis. In addition, co-treatment with bosentan and valsartan reduced $\alpha$-SMA and collagen IV expression in the UUO kidney compared to single treatments. These data show that bosentan and valsartan act complementarily, as co-treatment with both drugs had an additive protective effect against renal fibrosis.

\section{Disclosure Statement}

All authors declare that they have no conflict of interests to state. 


\section{Kidney \\ Blood Pressure Research}

\section{Acknowledgments}

This research was supported by Basic Science Research Program through the National Research Foundation of Korea(NRF) funded by the Ministry of Science, ICT \& Future Planning (NRF-2015R1C1A2A01055896). This research also was supported by a Clinical Research Institute grant (CMCDJ-P-2014-012) funded by The Catholic University of Korea Daejeon St. Mary's Hospital.

\section{References}

$1 \quad$ Klahr S, Schreiner G, Ichikawa I: The progression of renal disease. N Engl J Med 1988;318:1657-1666.

2 Klahr S, Morrissey J: Obstructive nephropathy and renal fibrosis. Am J Physiol Renal Physiol 2002;283:F861-875.

3 Bascands JL, Schanstra JP: Obstructive nephropathy: Insights from genetically engineered animals. Kidney Int 2005;68:925-937.

4 Kelly DJ, Zhang Y, Moe G, Naik G, Gilbert RE: Aliskiren, a novel renin inhibitor, is renoprotective in a model of advanced diabetic nephropathy in rats. Diabetologia 2007;50:2398-2404.

5 Pimentel JL, Jr, Montero A, Wang S, Yosipiv I, el-Dahr S, Martinez-Maldonado M: Sequential changes in renal expression of renin-angiotensin system genes in acute unilateral ureteral obstruction. Kidney Int 1995;48:1247-1253.

6 Qin J, Mei WJ, Xie YY, Huang L, Yuan QJ, Hu GY, Tao LJ, Peng ZZ: Fluorofenidone attenuates oxidative stress and renal fibrosis in obstructive nephropathy via blocking nox2 (gp91phox) expression and inhibiting erk/ mapk signaling pathway. Kidney Blood Press Res 2015;40:89-99.

7 Amann B, Tinzmann R, Angelkort B: Ace inhibitors improve diabetic nephropathy through suppression of renal mcp-1. Diabetes Care 2003;26:2421-2425.

8 Aki K, Shimizu A, Masuda Y, Kuwahara N, Arai T, Ishikawa A, Fujita E, Mii A, Natori Y, Fukunaga Y, Fukuda Y: Ang ii receptor blockade enhances anti-inflammatory macrophages in anti-glomerular basement membrane glomerulonephritis. Am J Physiol Renal Physiol 2010;298:F870-882.

9 Bichu P, Nistala R, Khan A, Sowers JR, Whaley-Connell A: Angiotensin receptor blockers for the reduction of proteinuria in diabetic patients with overt nephropathy: Results from the amadeo study. Vasc Health Risk Manag 2009;5:129-140.

10 Ohishi M, Takeya Y, Tatara Y, Yamamoto K, Onishi M, Maekawa Y, Kamide K, Rakugi H: Strong suppression of the renin-angiotensin system has a renal-protective effect in hypertensive patients: High-dose arb with ace inhibitor (hawaii) study. Hypertens Res 2010;33:1150-1154.

11 Karam H, Bruneval P, Clozel JP, Loffler BM, Bariety J, Clozel M: Role of endothelin in acute renal failure due to rhabdomyolysis in rats. J Pharmacol Exp Ther 1995;274:481-486.

12 Liss P, Carlsson PO, Nygren A, Palm F, Hansell P: Et-a receptor antagonist bq123 prevents radiocontrast media-induced renal medullary hypoxia. Acta Radiol 2003;44:111-117.

13 Lariviere R, Lebel M, Kingma I, Grose JH, Boucher D: Effects of losartan and captopril on endothelin-1 production in blood vessels and glomeruli of rats with reduced renal mass. Am J Hypertens 1998;11:989997.

14 Ritter C, Zhang S, Finch JL, Liapis H, Suarez E, Ferder L, Delmez J, Slatopolsky E: Cardiac and renal effects of atrasentan in combination with enalapril and paricalcitol in uremic rats. Kidney Blood Press Res 2014;39:340-352.

15 Erdogan H, Fadillioglu E, Emre MH: Protection from renal ischemia reperfusion injury by an endothelin-a receptor antagonist bq-123 in relation to nitric oxide production. Toxicology 2006;228:219-228.

16 Suzuki Y, Lopez-Franco O, Gomez-Garre D, Tejera N, Gomez-Guerrero C, Sugaya T, Bernal R, Blanco J, Ortega L, Egido J: Renal tubulointerstitial damage caused by persistent proteinuria is attenuated in at1-deficient mice: Role of endothelin-1. Am J Pathol 2001;159:1895-1904.

17 Bo Y, Chen YD, Li TD, Feng QZ: Endothelin-1 receptor blockade induces upregulation of renin-angiotensinaldosterone system expression in terms of blood pressure regulation. J Renin Angiotensin Aldosterone Syst 2010;11:119-123. 


\section{Kidney \\ Blood Pressure Research}

18 Gomez-Garre D, Largo R, Tejera N, Fortes J, Manzarbeitia F, Egido J: Activation of nf-kappab in tubular epithelial cells of rats with intense proteinuria: Role of angiotensin ii and endothelin-1. Hypertension 2001;37:1171-1178.

19 Miloradovic Z, Jerkic M, Jovovic D, Mihailovic-Stanojevic N, Milanovic JG, Stosic G, Markovic-Lipkovski J: Bosentan and losartan ameliorate acute renal failure associated with mild but not strong no blockade. Nephrol Dial Transplant 2007;22:2476-2484.

20 Zhang C, Chen YP, Dong HR, Qiu CB: [the therapeutic effects of bosentan and valsartan on renal interstitial fibrosis of chronic aristolochic acid nephropathy]. Zhonghua Yi Xue Za Zhi 2005;85:2601-2606.

21 Kelly DJ, Skinner SL, Gilbert RE, Cox AJ, Cooper ME, Wilkinson-Berka JL: Effects of endothelin or angiotensin ii receptor blockade on diabetes in the transgenic (mren-2)27 rat. Kidney Int 2000;57:18821894.

22 Cao Z, Cooper ME, Wu LL, Cox AJ, Jandeleit-Dahm K, Kelly DJ, Gilbert RE: Blockade of the renin-angiotensin and endothelin systems on progressive renal injury. Hypertension 2000;36:561-568.

23 Choi DE, Jeong JY, Lim BJ, Chang YK, Na KR, Shin YT, Lee KW: Aliskiren ameliorates renal inflammation and fibrosis induced by unilateral ureteral obstruction in mice. J Urol 2011;186:694-701.

24 Chang YK, Choi DE, Na KR, Lee SJ, Suh KS, Kim SY, Shin YT, Lee KW: Erythropoietin attenuates renal injury in an experimental model of rat unilateral ureteral obstruction via anti-inflammatory and anti-apoptotic effects. J Urol 2009;181:1434-1443.

25 Lee KW, Jeong JY, Lim BJ, Chang YK, Lee SJ, Na KR, Shin YT, Choi DE: Sildenafil attenuates renal injury in an experimental model of rat cisplatin-induced nephrotoxicity. Toxicology 2009;257:137-143.

26 Lehrke I, Waldherr R, Ritz E, Wagner J: Renal endothelin-1 and endothelin receptor type b expression in glomerular diseases with proteinuria. J Am Soc Nephrol 2001;12:2321-2329.

27 Moridaira K, Morrissey J, Fitzgerald M, Guo G, McCracken R, Tolley T, Klahr S: Ace inhibition increases expression of the etb receptor in kidneys of mice with unilateral obstruction. Am J Physiol Renal Physiol 2003;284:F209-217.

28 Vanourkova Z, Kramer HJ, Erbanova M, Backer A, Cervenka L, Huskova Z, Chabova VC, Tesar V, Dvorak P, Maly J, Vaneckova I: Endothelin receptor blockade does not affect blood pressure or angiotensin II levels in cyp1a1-ren-2 transgenic rats with acutely induced hypertension. Vascul Pharmacol 2009;50:194-199.

29 Choi DE, Jeong JY, Lim BJ, Chung S, Chang YK, Lee SJ, Na KR, Kim SY, Shin YT, Lee KW: Pretreatment of sildenafil attenuates ischemia-reperfusion renal injury in rats. Am J Physiol Renal Physiol 2009;297:F362370.

30 Peters H, Daig U, Martini S, Ruckert M, Schaper F, Liefeldt L, Kramer S, Neumayer HH: No mediates antifibrotic actions of l-arginine supplementation following induction of anti-thy1 glomerulonephritis. Kidney Int 2003;64:509-518.

31 Matsumura Y, Nishiura M, Deguchi S, Hashimoto N, Ogawa T, Seo R: Protective effect of fk409, a spontaneous nitric oxide releaser, on ischemic acute renal failure in rats. J Pharmacol Exp Ther 1998;287:1084-1091.

32 Boolell M, Allen MJ, Ballard SA, Gepi-Attee S, Muirhead GJ, Naylor AM, Osterloh IH, Gingell C: Sildenafil: An orally active type 5 cyclic gmp-specific phosphodiesterase inhibitor for the treatment of penile erectile dysfunction. Int J Impot Res 1996;8:47-52.

33 Ito K, Chen J, Seshan SV, Khodadadian JJ, Gallagher R, El Chaar M, Vaughan ED, Jr., Poppas DP, Felsen D: Dietary arginine supplementation attenuates renal damage after relief of unilateral ureteral obstruction in rats. Kidney Int 2005;68:515-528.

34 Ramzy D, Rao V, Tumiati LC, Xu N, Sheshgiri R, Miriuka S, Delgado DH, Ross HJ: Elevated endothelin-1 levels impair nitric oxide homeostasis through a PKC-dependent pathway. Circulation 2006;114:i319-326.

35 Dussaule JC, Tharaux PL, Boffa JJ, Fakhouri F, Ardaillou R, Chatziantoniou C: Mechanisms mediating the renal profibrotic actions of vasoactive peptides in transgenic mice. J Am Soc Nephrol 2000;11:S124-128.

36 Finch JL, Suarez EB, Husain K, Ferder L, Cardema MC, Glenn DJ, Gardner DG, Liapis H, Slatopolsky E: Effect of combining an ace inhibitor and a vdr activator on glomerulosclerosis, proteinuria, and renal oxidative stress in uremic rats. Am J Physiol Renal Physiol 2012;302:F141-149.

37 Manucha W, Oliveros L, Carrizo L, Seltzer A, Valles P: Losartan modulation on nos isoforms and cox-2 expression in early renal fibrogenesis in unilateral obstruction. Kidney Int 2004;65:2091-2107. 